\title{
El apoyo ciudadano a la acción extrajudicial de la política en Brasil, El Salvador y VenezuelaI
}

\section{Introducción}

En su primera declaración el vocero de la policía de Caracas dijo que los delincuentes habían fallecido en el enfrentamiento con los agentes. Era el mes de junio de 1996 y los vecinos había avisado a la policía que ocurría un atraco en la panadería. Un primer grupo de la policía había llegado al lugar del robo, enfrentó los ladrones y, en medio de la balacera, cayó muerta una mujer policía. Poco después llegaron los refuerzos y al percatarse que había todo un contingente de policías, los delincuentes se entregaron; así, salieron caminando del lugar y en esas condiciones fueron trasladados a las camionetas de la policía. Pocas horas después los entregaron muertos en un hospital público de la ciudad. La primera versión oficial resultaba bastante creible para quienes no se encontraban presentes; es normal que los delincuentes mueran en una refriega con las autoridades. El único gran problema fue que las cámaras de televisión, que habían llegado al lugar a tiempo, filmaron a los delincuentes caminando y entrando vivos en los carros de la policía.

1. Este artículo sirvió como base para una ponencia en el XXI Congreso de la Asociación Latinoamericana de Sociología, ALAS, Sao Paulo. Se fundamenta en una investigación multicéntrica, promovida y coordinada por la Organización Panamericana de la Salud: Proyecto ACTIVA, Normas culturales y Actitudes hacia la violencia, realizada en ocho ciudades de América Latina y España. Esta investigación recibió financiamiento local de la Secretaria de Saude de Brazil, de la Unión Europea y del CONICIT de Venezuela.

2. Laboratorio de Ciencias Sociales, LACSO, Universidad Central de Venezuela.

3. Istituto de Estudos da Religiao, ISER, Rio de Janeiro, Brasil.

4. Instituto Universitario de Opinión Pública IUDOP, Universidad Centroamericana, San Salvador, El Salvador. 
Esta historia no es ajena a muchos ciudadanos de América Latina. En tiempos más recientes los habitantes de San Salvador recuerdan al caso del joven Vilanova Velver, tanto como del joven Gaitán; los de Sao Paulo, los sucesos de Diadema y los de Rio de Janeiro al cabo Ferreira que en 1995 asesinó a un joven que había asaltado a una droguería sin saber que estaba siendo filmado y que su acción iría a ser transmitida por la televisión (Franco, 1997).

En Venezuela la Fiscalía de la República realizó en 1994 antejuicio a 202 funcionarios policiales acusados de homicidios y un organismo de defensa de los derechos humanos denunció que en 1995 se cometieron 126 homicidios por policías (PROVEA, 1995). En El Salvador, el $64.8 \%$ de las denuncias recibidas y registradas por la Procuraduría para la Defensa de los Derechos Humanos señalaban como responsables a miembros de la Policfa Nacional Civil por actos ilegales o arbitrarios en los procedimientos (IDHUCA,1997). En Río de Janeiro se ha hecho una estimativa provisoria de más de 300 muertos y más de 200 heridos en 1995; y en los primeros siete meses de 1997 se estimaba había superado los 200 muertos en acciones de la policía (ISER, 1997). Las razones de las muertes pueden ser múltiples, sin embargo, llama la atención que entre los policías hay regularmente más heridos que muertos, mientras que entre los delincuentes sucede lo contrario: hay más muertos que heridos; todo lo cual hace sospechar de ejecuciones realizadas por la policfa en sus operaciones (Cano, 1997).

\section{El monopolio de la violencia}

Uno de los rasgos más claros de la sociedad moderna es el monopolio de la violencia por parte del Estado. Toda asociación política tiene una forma de coacción física, todo poder ha estado asociado a la violencia como un modo de imponer su voluntad. Lo singular del Estado contemporáneo, es que "reclama para sí y con éxito el monopolio de la coacción física legítima" (Weber, 1977, II, 1056). La violencia física no es la única forma de acción del Estado, pero sí un medio especifico y legítimo de su actuación.

Al realizar esta operación, el Estado logra sustraer las acciones violentas y armadas del resto de la sociedad. La acción violenta que previamente la ejercían los particulares, los dueños de la tierra, los señores feudales o la Iglesia misma, es censurada y prohibida y el Estado se las reserva en nombre de la colectividad o del pacto que legítimamente asume representar. La justificación de este hecho se encuentra para algunos en la necesaria protección de los ciudadanos; el Estado es según Hobbes en su conocido Leviathan (1651) quien ha de proteger a las personas y a las propiedades contra la acción de otros individuos (Pérez Perdomo,1997), y para hacerlo así debe sacar del juego social las acciones violentas de los particulares y reservárselas para sí. Max Weber considera que es un medio por el cual logra construirse el mercado libre que ha de permitir un intercambio racional y legítimo, y no simplemente controlado por el poder y la 
fuerza (Weber,1977,I). Para otros autores, como Engels, esto es el reconocimiento que el conflicto entre las clases no ha podido resolverse solo, y que el Estado debe intervenir como un poder que aparente estar por encima de los intereses de clase y representar el interés general. Para otros autores este monopolio es fundamental para el establecimiento del capitalismo, pues su funcionamiento "no parece posible más que en la medida en que la represión física organizada no es ejercida directamente por los agentes en el dominio de las relaciones de producción sino que está reservada al Estado" (Poulantzas,1968, 294).

Pero esta violencia del Estado debe estar sometida a controles, debe ser controlada por la opinión publica, por la comunidad y para poder hacerlo debe estar normada, regulada por ciertos mandatos que restringen su ejecución a ciertas conductas y procedimientos que, por un lado le den legitimidad a la acción violenta del Estado, y, por el otro, protejan a los ciudadanos de los excesos o arbitrariedades de los funcionarios del Estado. La sociedad entiende que es muy riesgoso darle carta blanca de acción a un grupo organizado y armado como son los cuerpos de seguridad pública y/o las policías y por eso le coloca restricciones a su discreción de actuar. Es esto lo que conocemos como "estado de derecho".

Desde una perspectiva sociológica implica, además, una manera de actuar donde todos se encuentran sometidos a una norma, a una institución que se expresa en una ley abstracta y no personalizada. Es decir, que quienes ejercen y quienes reciben la violencia del Estado están controlados por la misma ley. Este proceso de abstracción permite que cuando se condene a alguien y se ejerza violencia no se haga nunca a nombre propio, sino a nombre de la ley. Esto hace que los actos de violencia del Estado - como la pena de muerte en los lugares donde existe formalmente - no sean nunca vistos como una venganza personal, sino como una acción abstracta justificada y legitimada por la ley.

\section{A. La acción extrajudicial de la policía}

Sin embargo la policía o las instituciones encargadas del orden social por parte del Estado pueden, en algunos momentos, actuar excediéndose en las facultades de actuación que les otorga la ley, y es eso lo que se conoce como acción extrajudicial. En este caso se violenta el estado de derecho y la policía desarrolla acciones que ignoran los derechos de los ciudadanos. No se trata aquí de cualquier tipo de acción ilegal de la policía; en la policía pueden darse casos de corrupción o simplemente delincuencia organizada que utiliza el uniforme y la protección policial- para sus acciones criminales. En el caso de la acción extrajudicial se refiere a las acciones y comportamientos ilegales de la policía que se ejecutan en el cumplimiento de su cometido legal, de sus objetivos institucionales. 
Por supuesto que en el caso de la acción extrajudicial, en muchos casos no es fácil diferenciar ni saber cuando el funcionario está simplemente excediéndose, por celo en el cumplimiento de sus funciones, y cuando se inicia una acción de uso privado del derecho a la violencia que el Estado le ha otorgado a ese funcionario. El mayor riesgo de la acción extrajudicial es que lo que pueda haberse iniciado como un celo de cumplimiento de sus deberes puede fácilmente derivar hacia organizaciones criminales que alquilen sus servicios a los grupos de poder o, inclusive, a los mismos criminales, bajo prácticas de limpieza social o de simple delincuencia.

Este hecho ocurre como una desviación de la policía y en la literatura se refiere como un proceso en el cual se da una separación entre los procesos de burocratización -que las vuelven impersonales-y de su sentido de comunidad. Las policías deben dar cuenta al mismo tiempo del poder constituido en la sociedad, expresado en las leyes y el derecho de las costumbres, una circunstancia abstracta pero fundamental; y de su sensibilidad hacia la comunidad en la cual trabajan y de la cual forman parte, una circunstancia personalizada que expresa el nivel de aceptabilidad que dicha policía tiene.

Teóricamente cuando la policía incumple la ley, debiera recibir un rechazo por parte de la comunidad y ésta debería usar los mecanismos establecidos en el mismo estado de derecho para reprender esas faltas. Pero una circunstancia especial acontece cuando la policía comete acciones extrajudiciales y empieza a tener apoyo por parte de la comunidad, pues se está violentando el estado de derecho. El problema es que, con frecuencia creciente en estos días, los policías o las personas investidas de autoridad en el área de la seguridad pública, perciben o interpretan las exigencias de la población para que sean más drásticos con la delincuencia, como una autorización valedera para la extralimitación de sus funciones en contra de la humanidad de los delincuentes. Ya no es raro en estos días, escuchar no sólo a agentes policiales sino también a funcionarios de alto rango, quejarse de leyes que "otorgan más protección a los delincuentes que a los ciudadanos honrados", en una expresión que muestra la facilidad para que las acciones de la polića puedan precipitarse en los senderos de la ilegalidad. Cuando las condiciones de inseguridad ciudadana se vuelven muy alarmantes para la población, la opinión pública - la comunidad misma - tiende a aflojar los controles con tal de contribuir al combate a la criminalidad y se crean las condiciones para que surjan las acciones extrajudiciales en las cuales la policía empieza a responder a la comunidad $\longrightarrow$ busca responder exclusivamente a ella- y no al marco legal.

\section{La investigación}

A fin de conocer sobre el apoyo que la población le da a las acciones extrajudiciales de la policía, se incluyeron un grupo de preguntas en una investi- 
gación que se ha llevado a cabo con apoyo de la Organización Panamericana de la Salud (OPS) en ciudades metropolitanas de América Latina. Este protocolo implicaba un diseño muestral similar y un cuestionario común para todas las ciudades participantes, así como un tiempo de recolección de la información similar (Briceño-León, 1997. Briceño-León et al.,1997). Este artículo presenta los resultados de las metrópolis de Caracas, Río de Janeiro y San Salvador; no está demás mencionar que, comparativamente, estas ciudades mostraron algunos de los porcentajes más altos en términos de victimización ciudadana a causa de la criminalidad.

\section{A. La muestra}

La investigación se realizó a partir de un protocolo común desarrollado por los investigadores y cuyo trabajo de campo consistió en una encuesta de preguntas cerradas y con escalas tipo Likert aplicada a personas entre 18 y 70 años de edad residentes en las áreas metropolitanas definidas. La encuesta se llevó a cabo en el hogar con una muestra estratificada polietápica y aleatorizada hasta lo interno del hogar. El número total de entrevistados fue de 1,298 en Caracas, 1,043 en Río de Janeiro y de 1,290 en San Salvador. La recolección de información tuvo una duración aproximada de dos meses por ciudad y se llevó a cabo entre julio y diciembre de 1996

\section{B. Las áreas de investigación}

Sobre un cuestionario de más de cien reactivos o ítemes, tres preguntas se realizaron para conocer el apoyo a la violencia extrajudicial y que cubrían diversos aspectos.

El primer lugar se indagó sobre el respeto a la libertad individual y al uso del estereotipo social del delincuente por parte de la policía y de la ciudadanía. En esta pregunta se busca reflejar una presunción que implica la segregación social, y todos los simbolismos que implica este hecho. Es el "etiquetamiento" (labelling) social que se asigna y se sugiere para conocer la reacción-respuesta del entrevistado. En este caso se preguntaba: "La policía tiene derecho a detener jóvenes que considere sospechosos por su aspecto físico".

Luego se trataba de conocer las reacciones que ante el principio de la inviolabilidad del hogar tenían las personas. Este principio se encuentra estipulado en las Constituciones de los países participantes en el estudio, por lo tanto, desde el punto de vista del estado de derecho para que la policía pueda ingresar en una casa debe tener una orden de cateo judicial. Se quería saber si se apoyaba el hecho que la policía entrara en una casa sin esta orden legal, es decir, lo hiciera extrajudicialmente. "La pregunta fue: La policía tiene derecho a invadir una casa 
sin orden de cateo para perseguir criminales".

Finalmente se intentó conocer la opinión de la población acerca del respeto a la integridad física de las personas detenidas, sobre todo en casos en los cuales se entra en conflicto con la posibilidad de que a través de medios ilegales, se pueda obtener mayor información acerca del delincuente: en particular, se planteaba acá la posibilidad de apoyo hacia las torturas aplicadas a las personas por los policías. La pregunta en cuestión fue: "En algunos casos se justifica que la policía torture a los sospechosos para obtener información". En los tres casos, las personas respondían mostrando su acuerdo o desacuerdo con la frase, en dos niveles de intensidad: muy (de acuerdo, desacuerdo) y algo.

Adicionalmente se quiso conocer acerca de la actuación de la policía, cómo las personas evaluaban a la policía y si habían recibido algún tipo de maltrato por parte de las autoridades.

Para esto se realizaron dos tipos de preguntas: "Basado en su experiencia y en lo que usted ha oído, cómo califica la eficiencia de la policía", y la otra pregunta sobre. "En los últimos doce meses la policía lo maltrató o golpeó a Usted". Con esto se pretendía poder examinar la relación que podía existir entre las respuestas dadas la acción extrajudicial y la acción de la policía en general, como experiencia personal y como percepción.

\section{IV.Los resultados del estudio}

Los resultados del apoyo a la acción extrajudicial presentados pueden observarse en los cuadros 1 y 2 . En el Cuadro 1 se muestran los resultados sumando las categorías muy de acuerdo y algo de acuerdo, para agrupar el apoyo dado a las acciones extrajudiciales. En el Cuadro 2 dos se presentan exclusivamente los datos de las respuestas "muy de acuerdo" para mostrar las diferencias en el énfasis mostrado en dicho apoyo. Estos datos fueron analizados de acuerdo con el sexo del entrevistado, su nivel educativo y su estrato social.

\section{Cuadro 1}

Apoyo a la acción extrajudicial de la policía algo de acuerdo y muy de acuerdo

(En porcentajes)

\begin{tabular}{lccc}
\hline Policía tiene derecho a: & Río de Janeiro & San Salvador & Caracas \\
\hline Invadir la casa sin orden judicial & 14.8 & 12.4 & 30.1 \\
Detener jóvenes por su aspecto físico & 16.5 & 26.8 & 26.5 \\
Torturar para obtener información & 12.5 & 16.1 & 17.7 \\
\hline
\end{tabular}


Sobre el detener a los jóvenes por su aspecto físico, el porcentaje es similar en Caracas y San Salvador para los acuerdos generales, una cuarta parte de la población la apoya en estas ciudades. Sin embargo, este porcentaje es más alto en San Salvador cuando los datos se refieren solo a quienes están muy de acuerdo (Cuadro2). Esto podría estar relacionado con el fenómeno creciente de las pandillas juveniles (llamadas "maras") en el caso salvadoreño - las cuales son un producto de la deportación de nacionales desde los Estados Unidos- y la demanda de la población por combatir ese problema. Al observar los datos por sexo se encuentran algunas diferencias entre las ciudades: en San Salvador no existe diferencia entre hombres y mujeres, en Caracas los hombres tendían a estar de acuerdo y las mujeres en desacuerdo; en Río de Janeiro sucedía los mismo, solo que la diferencia era más grande y los hombres duplican a las mujeres. Considerando el nivel educativo en las tres ciudades ocurre lo mismo: los menos educados son quienes apoyan la medida. Y, finalmente, desde la perspectiva de estrato social es similar que los pobres apoyan la acción de detener lo jóvenes, la única diferencia es que en Río de Janeiro la clase media también apoya la medida y en San Salvador la clase alta la rechaza.

\section{Cuadro 2}

Apoyo a la acción extrajudicial de la policía muy de acuerdo (En porcentajes)

\begin{tabular}{lccc}
\hline Policía tiene derecho a: & Río de Janeiro & San Salvador & Caracas \\
\hline Invadir la casa sin orden judicial & 7.5 & 6.3 & 22.3 \\
Detener jóvenes por su aspecto físico & 8.7 & 14.1 & 10.3 \\
Torturar para obtener información & 4.1 & 7.9 & 6.5 \\
\hline
\end{tabular}

En relación a la posibilidad de invadir una casa la población de Caracas está dos veces más de acuerdo que la población de Río de Janeiro y poco más del doble que en el caso de San Salvador. Estos datos no mostraron diferencia en relación al sexo del entrevistado, pero sí una importante diferencia en el nivel educativo y el estrato social. En Caracas los menos educados apoyan esta medida, en cambio en Río de Janeiro y en San Salvador son los más educados quienes tienden a apoyarla. Consistentemente en Río de Janeiro y en San Salvador la clase alta está de acuerdo y la clase baja en desacuerdo; en Caracas, no existe diferencia significativa entre los estratos.

Esto puede relacionarse con la existencia de regímenes dictatoriales, es posible que como en Venezuela no ha existido una dictadura desde hace cuarenta años, en Caracas no se tema a esto, pues el régimen de respeto a las libertades ha sido bastante grande y se piensa que esto se haría para detener delincuentes y no por algún otro propósito, o que pueda ser posible la arbitrariedad. Lo cual es 
diferente en el caso de Río de Janeiro o San Salvador, es decir, el apoyo disminuye conforme los regímenes dictatoriales han estado más cerca en el tiempo.

Sobre la tortura, vuelven a ser sorprendentes los resultados de Caracas quien tiene el porcentaje más alto, sin embargo, nuevamente, cuando se toma en consideración sólo quienes dijeron que estaban muy de acuerdo, San Salvador tiene un porcentaje superior a Caracas. Quienes apoyan la tortura provienen por igual de todas las clases sociales, se nota sin embargo en San Salvador un mayor apoyo por parte de la clase media y alta, lo que no sucede en Caracas o Río de Janeiro. Y en relación a la educación son los menos educados quienes apoyan la tortura.

Dos hechos llaman la atención, uno es que en San Salvador exista mayor apoyo al hecho que la policía torture a que invada las casas sin orden, lo cual es diferente en Caracas y en Río de Janeiro donde la tortura ocupa el último lugar en las acciones extrajudiciales apoyadas. En Caracas, a pesar que el apoyo a la tortura es alto, es mucho menor - casi la mitad- del apoyo dado a la acción de invadir a una casa. El razonamiento que puede existir en San Salvador, que viene de una dictadura y una guerra reciente, parece ser que la tortura se aplica a otros, mientras que la invasión a una casa puede afectarlo a uno, y esto puede marcar la diferencia. Acá es posible entrever los rezagos de concepciones ideológicas que durante la guerra salvadoreña tuvieron mucha vigencia. La tortura parece ser más permitida por aquel grupo menos expuesto a la misma y el cual justifica esas acciones como un medio de protección; en cambio, la irrupción al propio hogar es una medida menos lejana.

De manera general resulta sorprendente lo alto de los porcentajes a las acciones extrajudiciales dado en Caracas, que llegan a ser en algunos casos el doble del apoyo dado en Río de Janeiro. Una posible explicación está en la imagen de la policía y las experiencias de victimización, las cuales son peores y mayores en Caracas que en Río de Janeiro.

Con relación al maltrato policial, los datos son bastante similares en Río de Janeiro, Caracas o en San Salvador (Cuadro 3). Si bien entre estas ciudades no existen diferencias, hay que decir que al compararla con otras ciudades menos violentas de la región las cifras de estas tres ciudades - tan disímiles en varios aspectos- resultan entre cinco y siete veces mayores. No se encontraron asociaciones significativas estadísticamente entre victimización por parte de la policía y apoyo o rechazo a la acción extrajudicial.

El otro factor es la imagen de la policía, la cual si bien es similar entre Río de Janeiro y Caracas cuando se suman los que piensan que es mala o muy mala, es diferente y muy superior en Río de Janeiro las que consideran que es muy mala. La diferencia con los datos de San Salvador, que muestra los niveles más bajos de evaluación negativa, podría deberse a que la institución policial salvadoreña es muy joven, ya que solo nació a mediados de 1993 y como resultado 


\section{Cuadro 3 \\ Victimización por parte de la Policía \\ (En porcentajes)}

\begin{tabular}{llll}
\hline ACONTECIMIENTO & Río de Janeiro & San Salvador & Caracas \\
\hline $\begin{array}{l}\text { La policía lo maltrató o golpeo } \\
\text { en los últimos doce meses }\end{array}$ & 2.6 & 3.0 & 3.5 \\
\hline
\end{tabular}

\section{Cuadro 4 \\ Evaluación negativa de la Eficiencia de la Policía (En porcentajes)}

\begin{tabular}{llll}
\hline & \multicolumn{3}{l}{ ¿Cómo considera la efíciencia de la Polić́a? } \\
\cline { 2 - 4 } CIUDADES & Muy Mala & Mala & Regular \\
\hline Río de Janeiro & 11.1 & 15.7 & 44.4 \\
San Salvador & 3.6 & 14.4 & 42.8 \\
Caracas & 4.3 & 23.3 & 52.0 \\
\hline
\end{tabular}

de la firma de la paz. Estos resultados se cruzaron con el apoyo a la acción extrajudicial y se encontró una relación con la pregunta acerca de invadir una casa sin orden de cateo. En Caracas de un modo inconsistente desde el punto de vista de la acción racional, las personas que le daban apoyo a la acción extrajudicial de la policía eran los mismos que pensaban que la policía era regular o mala. En San Salvador por el contrario, y más de acuerdo a lo que se podría esperar, los que piensan que la policía es buena le dan apoyo a la medida y quienes piensan que es mala se lo niegan. En Río de Janeiro no hubo variaciones entre la evaluación que se hacía de la policía y las diferencias en el apoyo a la acción extrajudicial.

\section{Conclusiones}

Los resultados muestran que en las tres ciudades estudiadas existen proporciones significativas de población que apoyan las acciones extrajudiciales por parte de la policía. El hecho de que, al menos, uno de cada cinco ciudadanos vea con buenos ojos este tipo de acciones implica una presión notable a las actuaciones de las policías. Los datos señalan además que el apoyo al tipo de acciones es distinto para cada ciudad, muy probablemente a causa del contexto local.

Ahora bien, el apoyo que una parte de las comunidades nacionales le está dando a las acciones extrajudiciales de la policía constituye un cuestionamiento 
al estado de derecho muy superior al que representa la acción extrajudicial propiamente dicha. La policía puede excederse o actuar fuera de las atribuciones que le otorga la ley, pero esto no representaría un riesgo tan fuerte para la democracia y la legitimidad si no tuviera el apoyo ciudadano que lo impulsa y lo valida.

Se debe tomar en cuenta este apoyo por el impacto que puede tener en el comportamiento de la policía - no sólo en términos personales, sino sobre todo institucionales-, las políticas de las "premiaciones por bravura" (o valentía), como existe en Río de Janeiro, pueden tomar mayor fuerza institucional y darle aliento a la acción violenta injustificada de la policía. Con alicientes de este tipo, la policía no tendría temores de actuar violentamente en situaciones poco justificables pues podría asumir que tiene en contra la ley, pero a su favor el apoyo de la comunidad que la respalda.

Cuando se aborda este tema siempre puede surgir la respuesta de ¿por qué darle tanta importancia a los derechos de los delincuentes cuando éstos sistemáticamente violan los derechos de los ciudadanos?. La respuesta es unívoca desde el punto de vista ético, son ciudadanos igual que otros, no lo son menos. Pero hay también una respuesta profundamente práctica: la violencia es interacción social. Si la policía incrementa la violencia la delincuencia hará lo mismo, si el delincuente no cree tener una salida legal y no violenta, tomará el camino de una mayor violencia. Y la violencia genera más violencia.

Desde el punto de vista de las personas hay una inconsistencia global: ¿cómo es posible considerar que la policía es regular o mala y darle apoyo para que realice acciones extrajudiciales?. Esto pudiera interpretarse de dos maneras, puede ser que cuando los entrevistados responden a la pregunta del apoyo a la acción extrajudicial lo hacen pensando en el deber ser de la policía, en las funciones sociales que se le atribuyen a la policía con independencia de la verdadera policía que existe en esa ciudad. Otra interpretación, más extrema es que los entrevistados sí son consecuentes y realistas en ambas respuestas, solo que consideran que los policías no son eficientes ya que no son más violentos y no matan a más delincuentes, pues, si así lo fueran serían mejores policías.

Por otra parte, la acción extrajudicial puede ser un medio para reforzar el proceso de exclusión social. Detener a los jóvenes por su simple aspecto físico es un mecanismo de etiquetamiento (labelling) social, pues ocurre que a los excluidos sociales se les considera delincuentes por el simple hecho de ser pobres, mal vestidos, oscuros de piel o indios (Giddens, 1980). Y esto ocurre continuamente en la realidad, del total de detenidos por la policía metropolitana de Caracas en 1996, el $37 \%$ fueron de menores de edad para realizar "averiguaciones", es decir, por el solo hecho de ser jóvenes y pobres (Policía Metropolitana,1997). Esto, además de ser ilegal y segregacionista, puede convertirse en un factor de producción de delincuentes, pues, como bien lo demuestra la teoría del 
etiquetamiento, luego que se les etiqueta y se les trata como delincuentes, a las personas - y mucho más a los jóvenes - les es más fácil aceptarse y hacerse tales. Esto es algo que probablemente está sucediendo en El Salvador, donde la preocupación extrema por el fenómeno de las pandillas juveniles ha provocado que funcionarios gubernamentales se dediquen a atribuir el problema de la criminalidad a la juventud.

La situación es muy paradójica, pues la comunidad que debía decirle a la policía: "cuidado, respete los derechos humanos de los ciudadanos", está comenzando a decir lo contrario, y lo está haciendo por el miedo a la violencia y a la delincuencia (BID,1997; Guerrero,1996 ; Soarez e Carneiro,1996); además de que está comenzando a prescindir de los marcos legales-institucionales para resolver los conflictos y alcanzar los objetivos en términos de seguridad pública (IUDOP, 1996). El incremento notable en las tasas de homicidio que ocurren en todo el continente a fines de los años ochenta y comienzo de los noventa (Souza, 1994, Yunes y Rajs, 1994, OPS, 1996), hace que el miedo de las personas a ser víctimas de la violencia (Soarez,1996) los conduzca a apoyar medidas extralegales en un acto de desesperación por la angustia de lo que puede ocurrirles o por un deseo de venganza ante lo sufrido.

El apoyo ciudadano a la acción extrajudicial es muy preocupante pues puede conducir a salidas autoritarias de gobiemo y a mayor violencia en la sociedad. Con independencia del sentido de clase que pueda atribuírsele al estado de derecho, hoy en día su existencia representa una mayor protección para todos los ciudadanos y, en particular, para los débiles y los pobres, quienes no tienen acceso a los mecanismos de poder de la sociedad para hacer valer sus derechos y cuya única alternativa es el respeto por la ley que ha de ponerle límites a los poderosos sociales y a las instituciones que monopolizan legalmente la violencia. La defensa del estado de derecho es un mecanismo de construcción de ciudadanía que permite la posibilidad de construir un cambio social en paz.

Es necesario contrarrestar las tendencias de apoyo a la acción extrajudicial entre la ciudadanía y esto requiere de campañas de opinión pública contra este tipo de acción mostrando sus consecuencias negativas en el corto y mediano plazo. Pero, sin lugar a dudas, requiere al mismo tiempo, y sobre todo, del combate a la impunidad que permite los niveles de violencia; de una mejoría en los sistemas de protección ciudadana, requiere de la prevalencia del debido proceso y de una intervención policial más ajustada a derecho que haga sentir a los ciudadanos protegidos.

\section{Referencias bibliográficas}

BID. 1996 Hacia un enfoque integrado del desarrollo: ética,violencia y seguridad ciudadana. Washington,BID 
Briceño-León, R 1997 Buscando Explicaciones a la violencia, Espacio Abierto, Vol.6, N_ I, Enero - Abril, pp.45-69

Briceño-León, R , L. P. Carneiro,. Velez, J. M. Cruz,. Oviedo y A. McAlister,1997, Comparando Violencia y confianza en la Policía en América Latina, Revista Economi a y Ciencias Sociales,

Cano, I. 1997, Letalidad de Acao policial no Municipio de Rio de Janeiro, Ponencia en el XXI Congreso de la Asociación Latinoamericana de Sociología ALAS, Sao Paulo.

Franco Agudelo, Saul 1997,Violencia, Cidadania e Saúde Pública, en Equidade e Saúde, Contribucoes da Epidemiologia, R.B. Barata, M.L. Barreto, N. Almeida Filho, R.P. Veras (org.), Editora Fiocruz-Abrasco.

Giddens, A. 1980, Sociology, Cambridge, Polity Press.

Guerrero,R.1996, Epidemiología de la violencia: el caso Cali,Colombia. en BID: Hacia un enfoque integrado del desarrollo: ética, violencia y seguridad ciudadana. Washington, BID

IDHUCA, 1997, Instituto de Derechos Humanos de la UCA, Los derechos Humanos en El Salvador 1996. San Salvador, Publicaciones del IDHUCA.

ISER, 1997, Magnitude, Custos Economicos e Politicas de Controle da Violencia. Relatorio parcial submetido ao BID, Taller de Resultados, Cali, Colombia, Mayo.

IUDOP, 1996. Instituto Universitario de Opinión Pública. Las actitudes de los salvadoreños en torno a las leyes. Estudios Centroamericanos (ECA). 576, 905-920. San Salvador: UCA.

OPS, 1996. Violencia en las Américas: la pandemia social del siglo XX. Washington, OPS.

Pérez Perdomo, R y J.C. Navarro,1991 Seguridad Personal: un asalto al tema. Caracas, Ediciones IESA.

Policia Metropolitana, 1997, Diagnóstico de Seguridad en el área metropolitana de Caracas, Caracas, División de Planificación.

Poulantzas, 1968, Pouvoir politique et Classes Sociales, Paris, Maspero.

Provea, 1995, Situación de los derechos humanos en Venezuela. Informe anual, octubre 1994-septiembre 1995. Caracas

Soarez,L.E. ,1996, O inominavel, nosso medo en Violencia e Política no Rio de Janeiro, Rio de Janeiro, Relume Dumará-Iser, 59-64..

Soarez,L.E. y L.P.Carneiro, 1996, Os quatro nomes da violencia: um estudo sobre éticas populares e cultura polftica en Violencia e Política no Rio de Janeiro, Rio de Janeiro, Relume Dumará-Iser, 13-58

Souza. E.R. , 1994, Homicidios no Brasil: O grande Vilao de Saúde Pública na década de 80, en Cadernos de Saúde Pública, 10 (supl.1): 45-60.

Weber, M. 1971, Economia y Sociedad, México, Fondo de Cultura Económica.

Yunes,J. y D.Rajs, 1994, Tendencia de la mortalidad por causas violentas en la población general y entre los adolescentes y jovenes de la región de las Américas, Cadernos de Saúde Pública, 10 (supl.1): 88-125. 